\title{
Calibrated precise excision of uterine septum
}

\begin{abstract}
Uterine septum is the most common Mullerian abnormality. Previously the diagnosis was possible only by hysterosalpingography. Now it is possible to diagnose by ultrasonography, MRI and by hysteroscopy.

The route of resection is now Trans cervical, as it is minimally invasive. This avoids laparotomy and scar on the uterus. This also has short operative time, less blood loss, increased pregnancy rate and live birth rate and reduced abortion rate.

The extent of cut on the septum is decided at present by doing it under ultrasound control, ${ }^{1}$ observing the unification of the cavity and seeing both the osteii simultaneously, and easy movement of hysteroscope from one cornue to other, also it is done using intra uterine palpator $^{2}$ and gyneco -radiological technique. ${ }^{3}$

This is the deciding factor of completion of the procedure. This is arbitrary. If the cut is less, then this carries the risk of repeating the procedure and if cut is aggressive, it can damage the uterus. The advanced USG machines and MRI now provide better diagnosis and dimensions of the septum.

The distance between the tips of the open blades of the scissors is calculated, and then the size of septum is checked. This decides the number of cuts to be taken on the septum.

Septum consists of muscular and fibrous tissue. ${ }^{4}$ The distention of the uterus by the distending medium stretches the muscular part and fibrotic part. This will make the dissection lesser than needed, but the obstetric outcome does not change till the correction of the septum is $<1 \mathrm{~cm}$ than required. Under correcting is better than performing over corrections. ${ }^{5}$

Scissors are preferred to monopolar current in this study. This has the advantage of using isotonic medium; additionally if blood vessels are seen they can guide the plane of dissection. This technique reduces the need to re-confirm and prevents excess cut that may weaken the uterus that can pose a problem at the time of delivery.
\end{abstract}

Keywords: uterine septum, hysteroscopy

\section{Introduction}

Septum is the most common mullerian abnormality. This can cause reduced fertility, abortion, premature delivery, and malpresentation. Presently it is approached through cervix by hysteroscope. The resection is done either by monopolar current or scissors. It can also be done by LASER.

The septum is cut upto the extent that both osteii can be seen simultaneously and the visual impression that single cavity is seen and hysteroscope can be moved freely from one ostium to other. The septum is diagnosed by USG or MRI or an accidental finding during diagnostic hysteroscopy. The advanced USG image gives almost same dimensions as MRI.

The present study is an effort to make uterine cavity as normal as possible in a single intervention without the possibility of excess cut by measuring the dimensions of septum and measuring the length of cut by scissors.

\section{Methodology}

The permission of Institutional review board is taken. The informed consent was taken. Routine investigations like complete blood count, urine routine examination, random blood sugar was done. Cervical culture was done and patients were selected after the culture was negative. 3D sonography is done to decide the length and breadth [A to $\mathrm{B}$ and $\mathrm{A}$ to $\mathrm{C}$ and $\mathrm{B}$ to $\mathrm{C}$ distance is measured].

The patient is taken for septum resection once the cervical culture is negative for infection. The time selected is immediate post
Volume 9 Issue 5 - 2018

\section{Pandurang R Rotithor}

Obstetrics \& Gynecology, Sidhdheshwar Hospital and Research Center, India

Correspondence: Pandurang R Rotithor, Obstetrics \& Gynecology, Sidhdheshwar Hospital And Research Center Solapur, Nagar road, Wagholi, Pune, Maharashtra, India,Tel 912027050806, Email pandurangrotithor@gmail.com

Received: August 09, 20 I8 | Published:September 18, 2018 menstrual. Tablet Misoprostol is given in a dose of $600 \mathrm{mcg}$ orally the night before. Sufficient Dilatation is done to allow for smooth passage of hysteroscope. Scissors are used as they can be used with normal saline as distention medium while avoiding complications of electric burns.

The distance between the tips of open scissors is measured. This will be used to mark the points A, B. (Figure 1) based on sonography report. The number of cuts is calculated by dividing the length of the septum in the ultrasound report by the distance of the open blades of scissors. The mark is done by the scissors by the same number of times of the above figure. For example if the length of the septum is $1.5 \mathrm{cms}$, the open blade should go up for three times towards corneal end of septum.

The margin of error should be towards under correction than overcorrection, so that if required, the correction is made later. The first cut is taken at the point $C$ (Figure 2), where the white line is seen. Now, cut should join points $A$ and $C$ and point $B$ and $C$. The curvature should be a little convex on the lower side, [i.e. under correction].

The distance between point $\mathrm{A}$ and point $\mathrm{B}$ is measured with the help of open scissors blade. It should match the report. Due to a stretch on the cut septum there can be a small error in the actual correction (Figure 3).

This should match the report, if not a little more dissection is done till the distance between A and B approximately matches the report (Figure 4).

Now, one will be able to see the corrected cavity and both osteii 
simultaneously. Excision should be towards under correction rather than over correction (Figure 5).

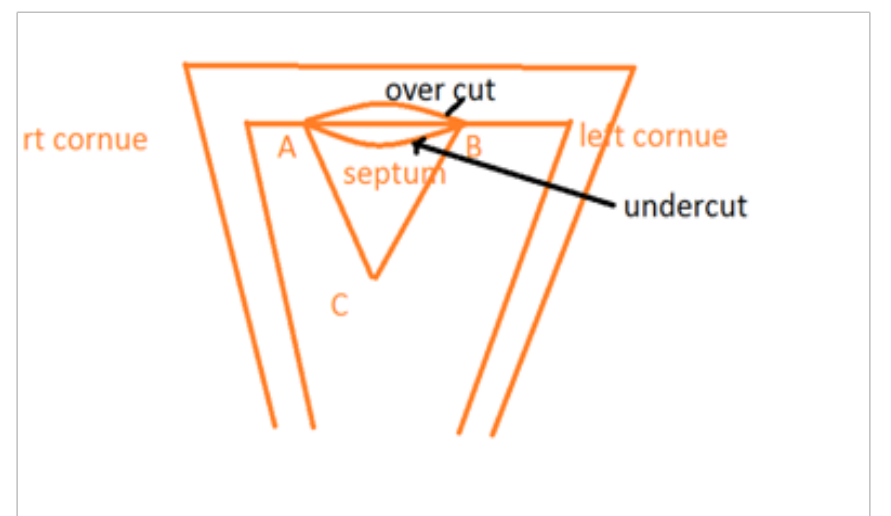

Figure I Diagram showing the points to be marked.

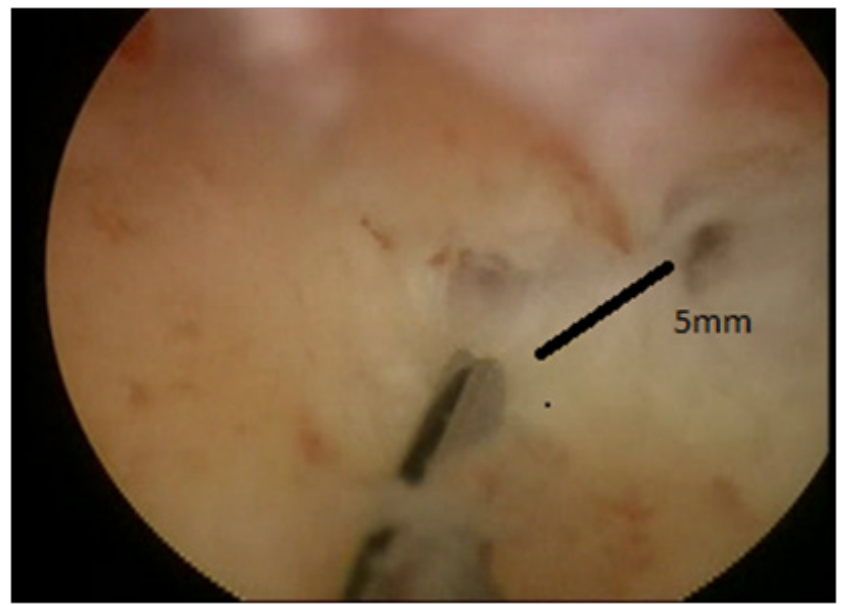

Figure 2 the marks of tips of scissors.

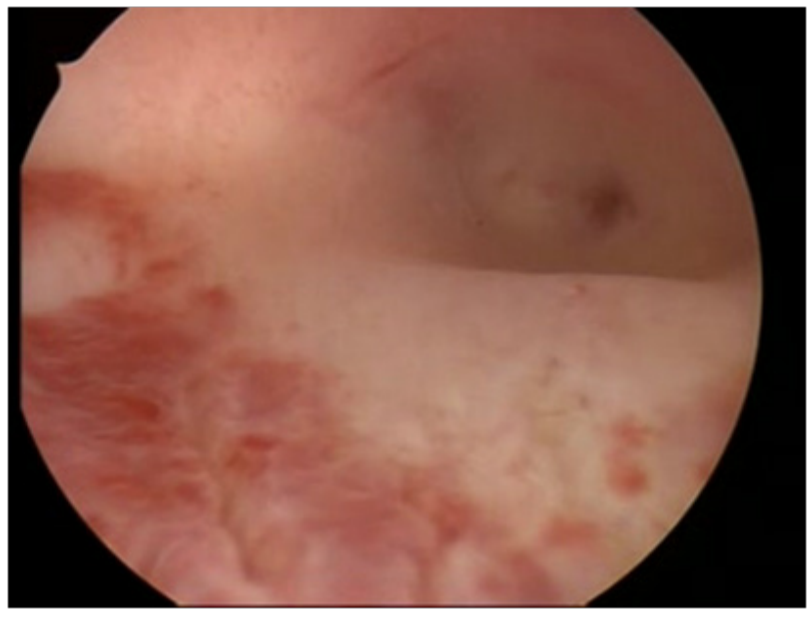

Figure 3 The point at which the first cut is to be taken.

The plane of dissection is better achieved by scissors than monopolar or bipolar current, as the bleeders can be seen very well when in wrong plane, then the cut should be done towards centre from the visible vessel. Balloon is placed in the uterus for 24 hours and the patient is put on estrogen for 21 days.

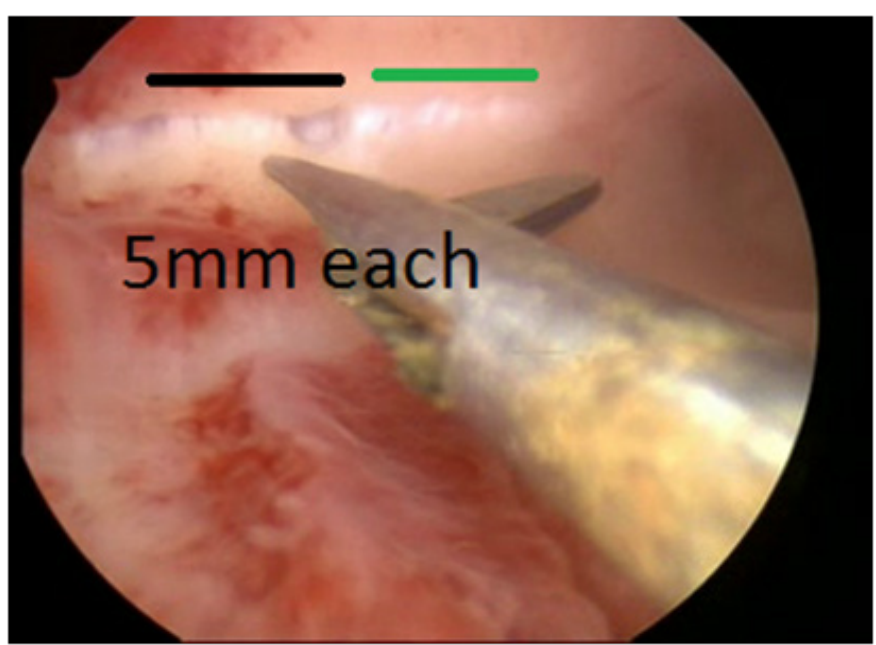

Figure 4 Measurement of point $A$ and point $B$.

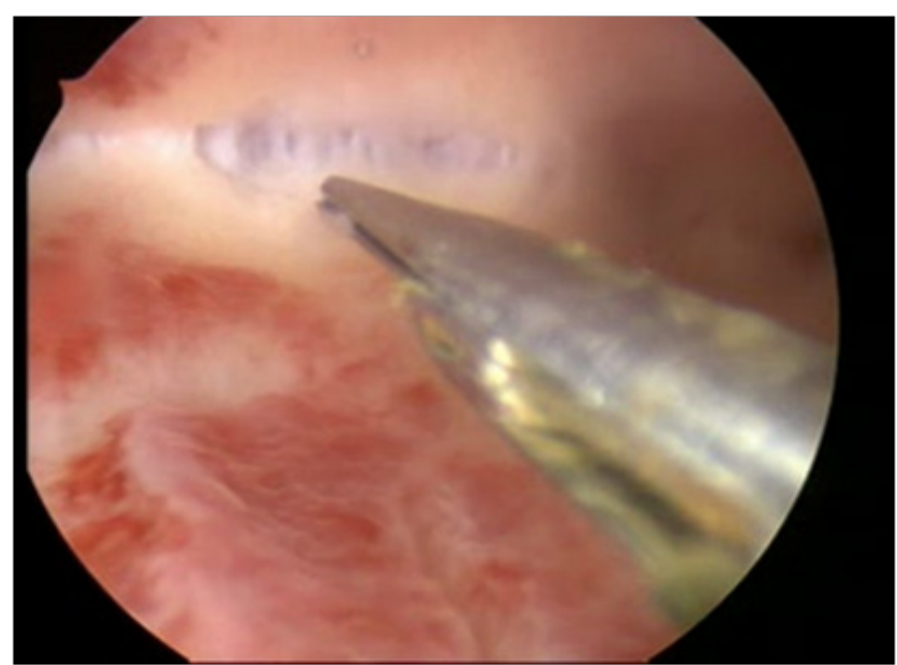

Figure $\mathbf{5}$ The corrected cavity and both osteii simultaneously.

\section{Results}

A total of 16 patients have undergone the procedure. 12 patients conceived and delivered full term baby. There was no difficulty in all the 12 cases. The post-operative USG is done after next menses. This is done to see the completion of the procedure. In all cases the resection was satisfactory without any need of repeat surgery.

\section{Discussion}

The extent of excision of septum is not well defined. Various methods are used for accomplishing the procedure.

It is done also under radiographic control. ${ }^{3}$

Seeing both osteii and ability to move the hysteroscope freely from one cornue to other is the criteria used by [R]. Ultrasound control during the procedure is used by many ${ }^{1}$ but it is cumbersome. Gynaecordiologic resection technique is done by some for completion of the procedure. ${ }^{3}$ The advantage is this does not need an operation theatre, but there is a risk of radiation. 
The intrauterine palpator makes accurate septal resection, but it needs special instruments. ${ }^{2}$ The measurement technique is simple, almost as accurate as the intrauterine palpator method.

This method comes closer to accurate dissection. The distance between point $\mathrm{A}$ and $\mathrm{B}$ is measured and this gives an almost exact resection of the septum. The method of using palpator is also fairly accurate but as compared to this method but you need separate instruments.

\section{Conclusion}

The present practice of correcting the uterine septum is arbitrary. The end point of cutting the septum is seeing both the osteii and ability to move the hysteroscope free lyare the deciding factors. The depth of septum resection is determined by the B ultrasonography or laparoscopic monitoring or by relative position of incised portion of septum and uterine horns. The additional markings to decide the extent of the cut helps to reduce the chance of overcutting the uterus and damage to muscular portion of uterus.

This also reduces the need to redo the procedure which saves time and cost. Another advantage of this method is using the scissors instead of monopolar knife helps to get correct plane. The vessels can be seen to guide the plane of dissection. This advantage is lost when you coagulate the vessel by current.

\section{Acknowledgments}

None.

\section{Conflicts of interest}

The author declares that they have no conflict of interest.

\section{References}

1. Ghirardi V, Bizzari N, Remorgida V, et al. Intraoperative Transcervical Ultrasonography for Hysteroscopic Metroplasty. J Minim Invasive gynecol. 2015;22(5):884-888.

2. Di Spiezio Sardo A, Zizolfi B, Bettocchi S, et al. Accuracy of Hysteroscopic metroplasty with combination of presurgical 3-Dimensional ultrasonography and a novel graduated intrauterine palpator a randomised controlled trial. J Minim Invasive Gynecol. 2016;23(4):557-663.

3. Karande VC, Gleicher N. Resection of uterine septum using gynecoradiological techniques. Hum Reprod. 1999;14(5):1226-1229.

4. Sparac V, Kupesic S, IIijas M, et al. Histologic architecture and vascularization of hysteroscopically excised intrauterine septa. $J A M$ assoc gyneco Laparosc. 2001;8(1):111-116.

5. Fedele L, Bianchi S, Marchini M, et al. Residual uterine septum of less than $1 \mathrm{~cm}$ after Hysteroscopic metroplasty does not impair reproductive outcome. Hum reprod. 1996;11(4):727-729.

6. Ata B, Nayot D, Nedelchev A, et al. Do measurments of uterine septum using three diamentional ultrasound, and magnetic resonance imaging agree? J Obstet Gynaecol Can. 2014;36(4):331-338. 